\title{
Regional Deposition: Targeting
}

\author{
Andrew R. Martin, PhD, PEng ${ }^{1, *}$
}

\begin{abstract}
Patterns of regional aerosol deposition within the lungs are known to vary in a predictable manner with a number of factors, most notably aerodynamic particle size and inhalation pattern. Targeting deposition involves the intentional manipulation of one or more of these factors to promote aerosol deposition in certain locations within the respiratory tract. This section will begin by exploring existing evidence supporting the need to target regional deposition. Thereafter, various approaches to targeting will be introduced. In addition to control of aerodynamic particle size and inhalation pattern, a collection of approaches are available through which to passively target deposition to more central or peripheral lung regions. These include the delivery of short aerosol boluses at prescribed time points in inhalation, control of transient hygroscopic aerosol size changes during transport through the respiratory tract, and use of alternative carrier gas mixtures such as helium/oxygen mixtures. Comparatively, targeting aerosol deposition locally to very precise, spatially-defined lung regions is in its infancy. Early, exploratory techniques used for local targeting will be described. The continued evolution of deposition targeting towards ever more specific locations within the lungs is required to explore fundamental research questions in aerosol medicine: namely, how precise does targeting need to be before additional refinement fails to produce appreciably different therapeutic effects, and which nascent applications of aerosols in medicine might benefit from more selective regional targeting?
\end{abstract}

Keywords: aerodynamic size, central deposition, deposition pattern, inhalation drug delivery, inhalation pattern, particle size, peripheral deposition, targeted drug delivery

\section{Introduction}

$\mathbf{I}^{\mathrm{N}}$ N GENERAL TERMS, targeted drug delivery refers to any method used to increase drug concentration at certain sites within the body relative to other sites. The aim is straightforward: to increase the therapeutic effect of the drug at its targeted location or locations, while reducing side effects that arise from accumulation of drug in non-targeted tissues and organs. For conventional routes of administration, numerous delivery vehicles have been explored to enable targeting, including polymer chains, micelles, liposomes, and nanoparticulate carriers. $^{(1,2)}$ Targeting is often passive, where the size of circulating, drug-carrying molecules or particles is tuned to promote uptake into selected tissues with increased vascular permeability, as occurs for many tumors and in infarcted areas. ${ }^{(2)}$ Alternatively, targeting may be active, where the drug carrier is bound with a molecule recognizable by cell surface receptors in the target tissue. ${ }^{(1)}$ These forms of molecular targeting most certainly have relevance for aerosol drug delivery; however, in the context of regional deposition of aerosols in the lungs, the term targeting often means something else - namely, the intentional manipulation of regional deposition patterns to promote aerosol deposition in certain locations within the respiratory tract. This definition of targeting aerosol deposition will be the focus of the present section.

At a high level, administering therapeutic agents directly to the lungs through inhalation is a form of targeting in and of itself. Improved targeting of the lung may be achieved by reducing upper airways deposition, such that a greater fraction of the emitted dose reaches the lungs. ${ }^{(3-8)}$ Alternatively, some delivery systems intentionally target deposition in the upper airways, as is the case for local nasal delivery. Taken a step further, many inhaled therapies are developed under the assumption that the regional deposition pattern within the lungs is an important determinant of their efficacy. For example, both asthma and chronic obstructive

Reprinted from The International Society for Aerosols in Medicine Textbook: https://www.isam.org/awards.

${ }^{1}$ 10-324 Donadeo Innovation Center for Engineering, University of Alberta, Alberta, Canada.

*Member of ISAM. 
pulmonary disease (COPD) have been characterized by inflammation affecting the small, peripheral airways; therefore, targeting deposition of inhaled corticosteroids to small airways may improve treatment. In other circumstances, where the inhalation route is used for systemic delivery, targeting deposition to the alveolar lung region may be used to enhance drug absorption efficiency.

This section will begin by investigating existing evidence supporting the need to target regional deposition. Thereafter, various approaches to targeting will be introduced. In categorizing these approaches, we will take the liberty of borrowing loosely from the terminology introduced above for drug targeting techniques in general. Accordingly, passive aerosol targeting is defined to encompass those techniques that do not require specific knowledge of the spatial location of target sites but involve manipulation of one or more factors to promote deposition in certain anatomical or physiological regions (for example, in conducting airways or in poorly ventilated lung regions). Conversely, local aerosol targeting will be used herein to refer to techniques that aim to specifically target deposition to one or more spatially defined locations within the respiratory tract.

Figure 1 lists approaches to targeting described in this section and sorts these between passive and local categories. Many of the techniques to be discussed draw from our evolving understanding of various mechanisms influencing regional deposition patterns in the respiratory tract, which have been introduced earlier in this text. Similarly, methods for assessing deposition targeting through imaging and pharmacokinetics will not be presented in detail here but are described elsewhere in the text.

\section{Regional Lung Deposition and Therapeutic Effect}

For topical treatment of airway diseases, the notion that efficacy will be improved by accurately targeting deposition of therapeutic aerosols to areas of inflammation, hyperresponsiveness, or obstruction seems so intuitive that it is often taken for granted. Yet, direct evidence to support this assertion is limited. The shortage of evidence can be understood in part by considering the complexity of obtaining, in a single study, accurate information describing, a) the location of sites to be targeted, b) the regional distribution of deposited drug within the lungs, and c) some measure of the drug's effect. Adding to the puzzle, numerous mechanisms act to transport and relocate drug or drug-containing particles after they have deposited in the lungs, including dissolution, absorption, liquid spreading over lung surfaces, mucociliary transport, and phagocytosis. ${ }^{(9)}$

Given these challenges, it is not surprising that some early studies which broadly targeted central or peripheral airways using polydisperse aerosols found little or no evidence linking regional deposition patterns within the lungs to therapeutic effects. ${ }^{(10,11)}$ More recently, Usmani and colleagues ${ }^{(12)}$ targeted deposition of radiolabeled, monodisperse albuterol aerosols to the conducting airways of asthmatics, on the basis that smooth muscle linked to airway hyperresponsiveness is predominantly located in the conducting airways. Indeed, combinations of particle size and inhalation flow rate used to promote central airway deposition were associated with greater bronchodilation. ${ }^{(12)}$ Associations between monodisperse pharmaceutical aerosol size and post-treatment lung function in asthmatics were also observed by Zanen and coworkers, ${ }^{(13,14)}$ although expected variation in deposition pattern with particle size was not confirmed by measurement. For maintenance treatment of cystic fibrosis, Bakker and colleagues ${ }^{(15)}$ reported a larger treatment response to inhaled dornase alfa when targeting small airway deposition versus large airway deposition, provided children were adherent to treatment. In contrast, the same group did not detect a significant difference in response between small versus large airway targeting of dornase alfa to cystic fibrosis patients experiencing respiratory tract exacerbations, although in this case dosing to the small airways may have been suboptimal. ${ }^{(16)}$

When the inhalation route is employed for systemic drug delivery, it is widely accepted that deposition in the alveolar region, with its combination of large surface, thin barrier to absorption, and slow clearance, should be targeted. ${ }^{(9,17,18)}$ Yet, as reviewed by Patton and Byron, ${ }^{(18)}$ even in the case of systemic delivery, exact target sites for optimal pulmonary absorption are not entirely clear and can vary depending on the size, hydrophobicity or hydrophilicity, and in some cases receptor location, of the molecule to be absorbed. For example, whereas deep lung deposition appears to be optimal for small peptides and insulin, large immunoglobulins of class $\mathrm{IgG}$ might be best targeted to the central airways, where receptormediated transport across the epithelium occurs. ${ }^{(18,19)}$

While the studies cited above employed various techniques to broadly and passively target central or peripheral lung deposition, more precise targeting may be useful in the treatment of diseases that are localized to one or more lung lobes or segments. Such need may arise, for example, in treating recurrent infections of the lungs of AIDS or cystic fibrosis patients, ${ }^{(20)}$ or for several types of lung cancer. ${ }^{(21)}$ In the latter case, aerosol delivery of chemotherapeutic agents carries the tantalizing promise of increasing drug concentrations in the lungs while minimizing systemic toxicities. However, significant concerns remain regarding pulmonary toxicity. ${ }^{(22)}$ Where tumors are confined to specific lung lobes, broadly delivering aerosol to all lobes in parallel is likely not an optimal strategy. In healthy canines, Selting and colleagues demonstrated that targeting chemotherapy to a single lung lobe using an intracorporeal nebulizing catheter resulted in high local drug concentrations in the targeted lobe. ${ }^{(23,24)}$ Furthermore, evidence of pulmonary toxicity was confined to that same lobe. Should this added precision in targeting translate to improved efficacy in tumor-bearing animals, delivery of inhaled chemotherapy could potentially present a nascent clinical application for local aerosol targeting.

On the whole, this discussion highlights the myriad applications of medical aerosols in current use or in development. Established approaches to passive aerosol targeting, such as controlling the size of inhaled particles or encouraging a certain patient breathing maneuver, have been crucial in developing existing products and in enabling researchers to better explore the extent to which regional deposition influences therapeutic effects. Whether or not the considerable effort required to more precisely identify and target optimal deposition sites within the lungs is warranted for a given therapeutic application will need to be evaluated on a case-by-case basis. Likely in most circumstances, a law of diminishing marginal returns will apply, whereby increasing the precision of targeting beyond a certain limit fails to deliver any appreciable added benefit. Has this limit 

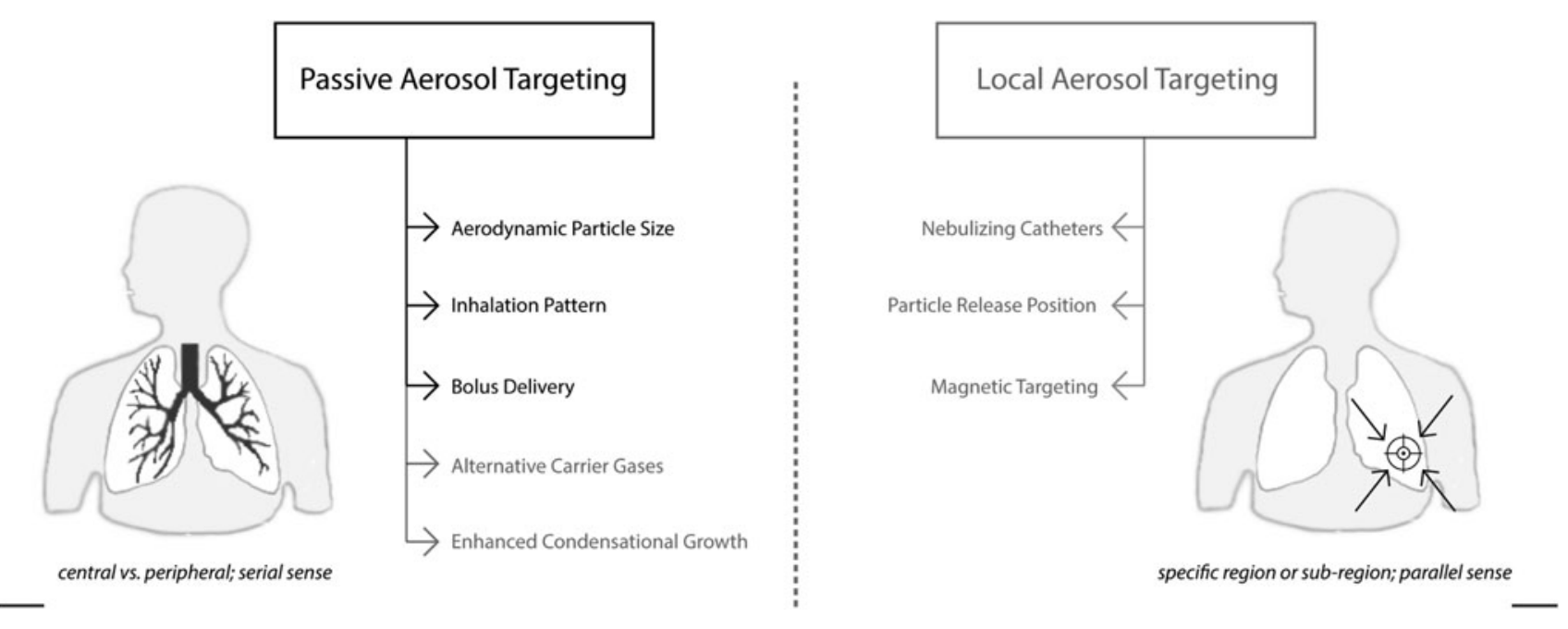

FIG. 1. Methods for targeting regional aerosol deposition within the lungs. Established techniques appear in black, whereas exploratory techniques appear in grey.

already been reached for some inhaled therapies (e.g., salbutamol) already widely used for many decades? Is a lack of sufficiently precise targeting hindering development of some new therapies? These are important questions, and the increasing availability of techniques for targeting deposition to ever more specific locations within the lungs represents one step towards an answer.

In what follows below, various approaches and techniques used to target regional deposition within the lungs are reviewed. Some of these approaches are already widely used in development of inhaled therapies, whereas others have only recently appeared in academic research. Effort has been made to present each approach descriptively and objectively rather than to recommend one approach over another. Clearly, the choice of targeting approach will depend on the given therapeutic application, and in some cases, effective regional targeting may well require a combination of several techniques.

\section{Passive Aerosol Targeting}

As defined above, passive aerosol targeting does not require detailed a priori knowledge of the spatial location of target sites but rather involves manipulation of one or more factors to promote deposition in certain anatomical or physiological regions. Accordingly, these approaches tend to broadly shift deposition between lung regions, for example between the central and peripheral lung, rather than to target specific, spatially-located airways or collections of airways. Given that such precise target locations are very rarely known, passive aerosol targeting provides a practical method for optimizing aerosol drug delivery. Some of the passive targeting techniques listed below will be familiar, having been adopted in the design of inhalers and"smart" delivery controllers currently on the market; others are exploratory, but show considerable promise in their ability to redistribute deposition patterns within the lungs.

\section{Aerodynamic particle size}

Inertial impaction is the predominant mechanism of aerosol deposition in the upper and central airways; therefore, factors that influence inertial impaction efficiencies can strongly affect the fraction of aerosol that deposits in the upper and central airways versus that which penetrates to the peripheral lung. As reviewed in earlier sections of this textbook, the aerodynamic particle size is a key determinant of inertial impaction efficiency. Targeting deposition to the central or peripheral lung using larger or smaller aerodynamic particle sizes, respectively, is wellentrenched in aerosol medicine. However, it must be understood that the refinement in regional targeting that can be achieved via aerodynamic size alone is coarse and highly variable between patients with different underlying airway geometries, varying manifestation of disease and who might be inhaling at different flow rates or otherwise breathing with different patterns.

Figure 2 displays traces of average fast- and slowcleared deposition fractions compiled by Stahlhofen and coworkers ${ }^{(25)}$ in healthy human subjects during tidal, oral breathing. Some caveats aside, the fast-cleared fraction can be considered to be indicative of deposition in the central, tracheobronchial airways, whereas the slow-cleared fraction can be considered to represent deposition in the peripheral, alveolar lung region. The influence of aerodynamic particle size on regional deposition in Figure 2 is clear but so too is a considerable overlap between broad peaks defining central and peripheral lung deposition. For polydisperse size distributions commonly generated from inhalers and nebulizers, the demarcation between central and peripheral deposition will be further blurred. While aerodynamic particle size is no doubt a critical factor influencing regional deposition patterns, additional variables must be manipulated in order to more precisely target deposition within the lungs.

\section{Inhalation pattern}

Along with particle size, the inhalation flow rate has long been identified as an important determinant of deposition in the upper and central airways. All else being equal, the probability that a particle of given aerodynamic size will impact on airway walls increases directly with the inhalation flow rate. 


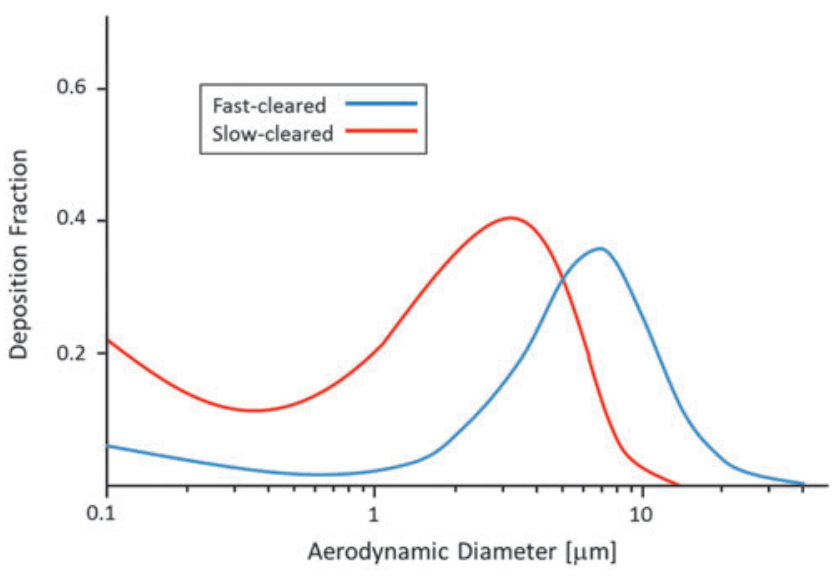

FIG. 2. Broad overlap in deposition fractions between central (fast-cleared) and peripheral (slow-cleared) lung regions is observed when plotted against particle aerodynamic size. The curves represent average deposition fractions compiled by Stahlhofen and coworkers ${ }^{(25)}$ in healthy human subjects during tidal, oral breathing.

Accordingly, instructing subjects or patients to inhale at low flow rates has been shown to shift deposition from more proximal to more distal airways. ${ }^{(26-28)}$ For commercial singlebreath inhalers, however, the potential use of low inhalation flow rates to reduce inertial impaction is often compromised by the requirement of reasonably high flow rates needed to fluidize and deaggregate dry powders, ${ }^{(29,30)}$ or to entrain highspeed droplet clouds emitted from pressurized metered-dose inhalers. ${ }^{(31-33)}$ Independent of the inhalation flow rate, patients are commonly instructed to hold their breath for several seconds after inhaling so as to allow aerosols more time to settle under the influence of gravity in peripheral airways. ${ }^{(34)}$ This is a logical recommendation in principle, but often not followed by patients. In practice, a goal in the design of most singlebreath inhalers is to achieve a repeatable lung dose independent of the patient inhalation maneuver. ${ }^{(35)}$

In contrast, for nebulizers commonly used to deliver aerosols during tidal breathing, there exists greater opportunity to manipulate regional deposition patterns by varying the inhalation pattern. Fittingly, devices have been developed to guide or control patient breathing through nebulizers. ${ }^{(36,37)}$ Figure 3 highlights the considerable variation in central and peripheral deposition of a monodisperse, $3 \mu \mathrm{m}$, aerosol achieved through variation of inhalation flow rate and inhaled volume. ${ }^{(38)}$ The latter is an important factor in determining the fraction of inhaled aerosol that is transported through the central, conducting airways and made available to deposit in the peripheral lung.

Figure 3 can be viewed in different ways: on the one hand, where inhalation pattern can be controlled, it provides a useful tool for targeting central or peripheral regions. That said, it must be understood that both central and peripheral deposition fractions depend on an individual subject's lung volume and airway geometry, thus the effectiveness of any prescribed inhalation pattern (or particle size) in achieving regional targeting will be variable between subjects. Accordingly, definition of a single set of targeting parameters suitable for all subjects within a large population is not possible. ${ }^{(39)}$ On the other hand, where inhalation pattern is allowed to vary, Figure 3 highlights just how variable deposition patterns may be, even when aerodynamic particle size is tightly controlled. Furthermore, because targeting deposition through control of inhalation patterns requires cooperation of the patient, these techniques benefit from the inclusion of means to monitor patient adherence to prescribed settings for use. ${ }^{(36,37)}$

\section{Bolus delivery}

To further improve passive aerosol targeting that can be achieved by varying aerodynamic particle size and inhalation pattern, bolus delivery of aerosol during only a portion of the total inhalation time has been employed. ${ }^{(37)}$ Limiting aerosol administration to the early portion of inhalation allows a greater fraction of inhaled fine particles to transit the conducting upper and central airways to reach peripheral lung regions. Conversely, administering a bolus of larger aerosol particles later in the breath may be used to limit their penetration into the peripheral airways, thereby effectively targeting central airways at an approximate volumetric lung depth. A secondary effect of bolus delivery may be in preferential administration of aerosol to well- versus poorly-ventilated lung regions, similar to effects described for pulsed delivery of medical gases. ${ }^{(40)}$ Poorly-ventilated regions tend to receive a greater fraction of ventilation late in inhalation, as the driving pressure gradient between the airway opening and other, better-ventilated regions decreases during filling.

As bolus delivery relies heavily on the timing of aerosol administration during the breath, it is very often linked with techniques used to control or guide patient inhalation patterns. For example, Clark and coworkers ${ }^{(41)}$ demonstrated that a bolus of coarse aerosol could be efficiently delivered to the peripheral lung using an initial low inhalation flow rate $(8 \mathrm{~L} / \mathrm{min})$ during bolus delivery followed by a larger flow rate $(30 \mathrm{~L} / \mathrm{min})$ for the remainder of inhalation, the intent being to maintain low flow only so long as the bolus transited the central, conducting airways. In general, just as for targeting by inhalation pattern alone, bolus techniques that rely on combination with controlled patient inhalation patterns require careful monitoring of those patterns to ensure that selected regions are indeed targeted.

\section{Helium/oxygen}

The use of helium/oxygen gas mixtures (commonly known as heliox) as alternative carrier gases for aerosol delivery has been investigated and will be described in detail in a separate section of this chapter. In the context of targeting regional deposition, it suffices here to comment that heliox offers two potential advantages for targeting small airways: first, inhaling particles with heliox in place of air tends to reduce deposition in upper and central airways such that a greater fraction of particles deposits in peripheral lung regions ${ }^{(42-47)}$; second, and more speculatively, increased ventilation of obstructed lung regions with heliox may transport more particles into those regions, thereby increasing the fraction of particles available to deposit in obstructed airways. ${ }^{(46,47)}$ An example of the former effect is provided in Figure 4. For the asthmatic patient shown, using heliox in place of air dramatically shifted deposition from central to peripheral airways. ${ }^{(46)}$ In general terms, this very 

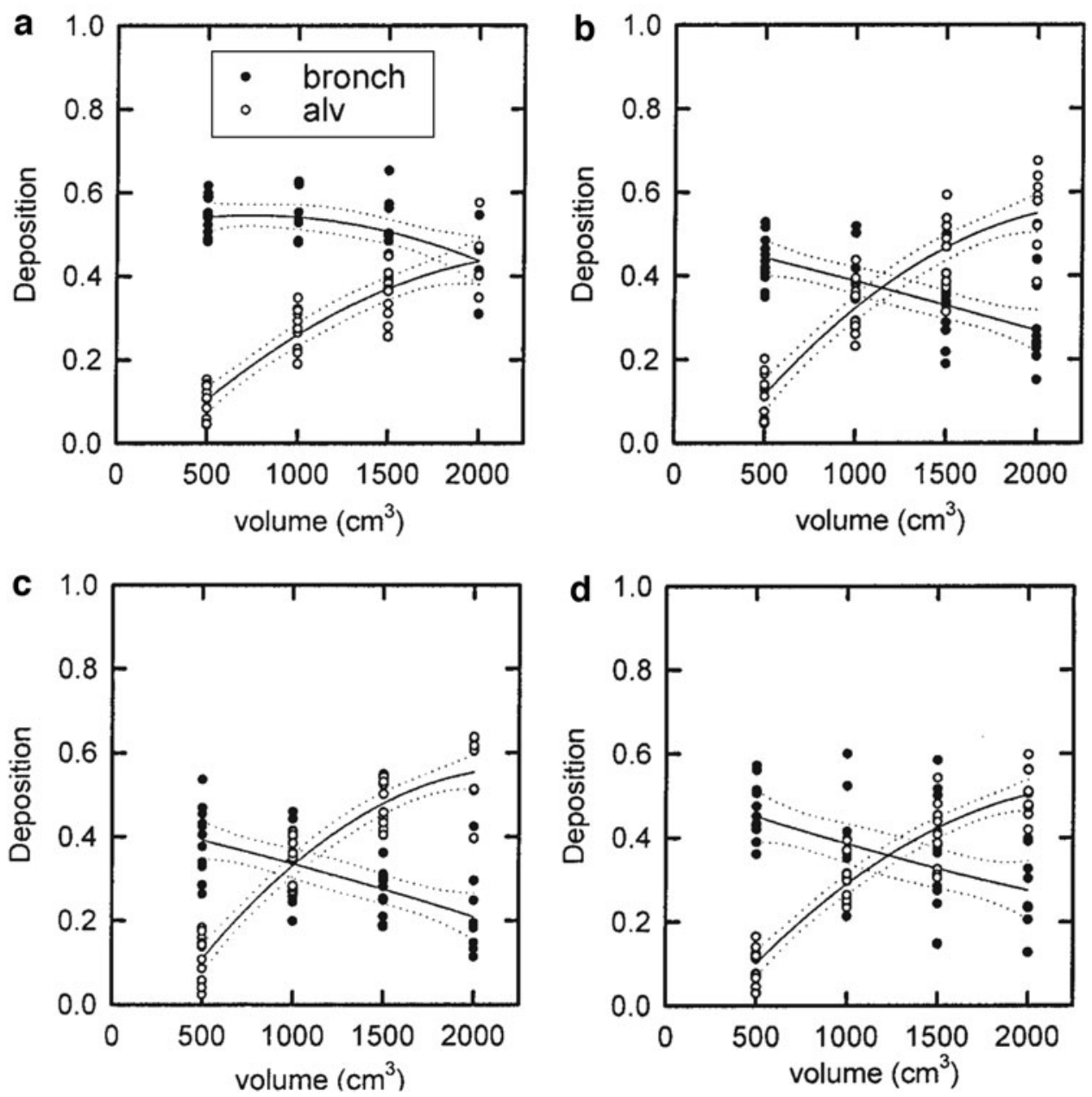

FIG. 3. Central (dark circles) and peripheral (white circles) deposition fractions plotted against inhaled volume for $3 \mu \mathrm{m}$ aerodynamic diameter particles inhaled at flow rates of, (a) $100 \mathrm{~cm}^{3} / \mathrm{s}$, (b) $250 \mathrm{~cm}^{3} / \mathrm{s}$, (c) $500 \mathrm{~cm}^{3} / \mathrm{s}$, and (d) $750 \mathrm{~cm}^{3} / \mathrm{s}$. Solid lines are best-fit polynomial curves. Dotted lines show the confidence intervals for the fitted curves. Reproduced from Brand and colleagues with permission from Mary Ann Liebert. ${ }^{(38)}$

likely resulted from effects of gas density and viscosity on fluid and particle motion within airways remodeled by disease. Potentially confounding effects of gas heat- and masstransfer properties on hygroscopic aerosol size changes were limited by using a high concentration aerosol. ${ }^{(48,49)}$ It must be noted that a second asthmatic patient saw next to no difference in deposition pattern breathing heliox versus air. Given that particle sizes and patient breathing patterns were carefully controlled, it was speculated that this intersubject variability resulted from variation in morphological changes associated with disease. ${ }^{(46)}$ In view of the complexities of incorporating heliox into aerosol delivery systems, improved means to identify individual patients or patient subgroups for which the gas will strongly influence regional deposition are required.

\section{Enhanced condensational growth}

As described above, and elsewhere throughout this text, aerodynamic particle size is a key determinant of regional deposition patterns. Unlike stable aerosols selected for use in many laboratory deposition experiments, aerosol particles or droplets inhaled from delivery devices often undergo transient size changes that arise due to evaporation of propellants or solvents and/or to condensation of water vapor from the humid environment of the respiratory tract. Longest, Hindle, and colleagues ${ }^{(8,50,51)}$ have proposed to control such effects so as to advantageously adjust aerosol sizes in transit, thereby promoting deposition at a desired lung depth. Similarly, hygroscopic size changes occurring for two approaches to transient size manipulation have recently been analyzed by Javaheri and Finlay. ${ }^{(52)}$ Put simply, inhaled submicrometer particles that grow by condensation as they travel from upper to central to peripheral airways will tend to deposit in distal regions while avoiding upstream filtering in more proximal regions. These approaches have the potential to improve the precision of targeting to central or peripheral lung regions that can be achieved through variation of stable particle size. Their implementation may involve development of novel administration devices to carefully control aerosol size, gas temperature, and water vapor content delivered to the upper airways ${ }^{(53)}$; alternatively these principles 

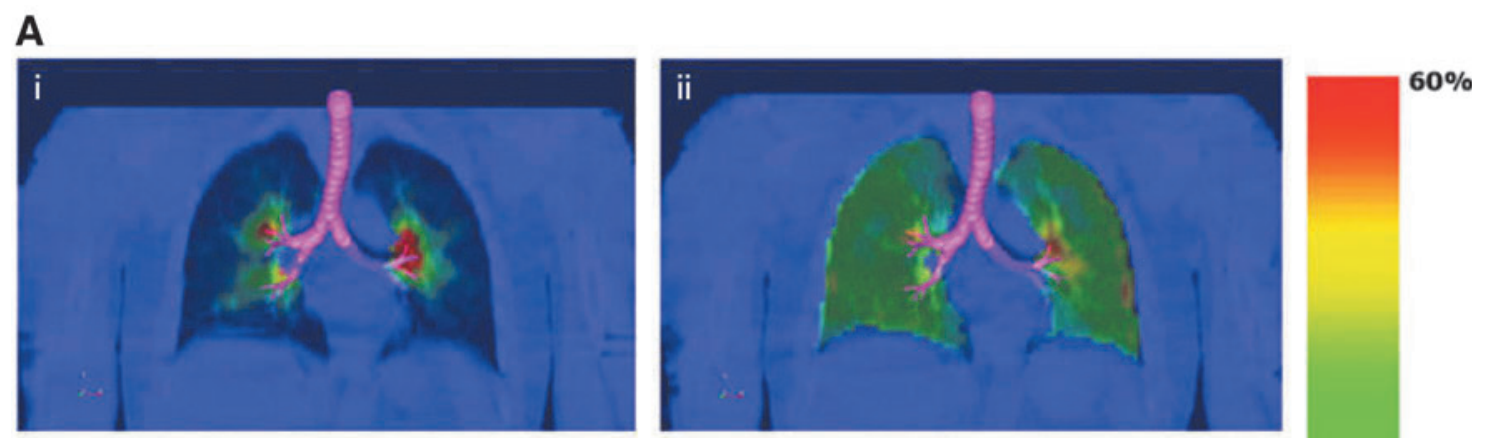

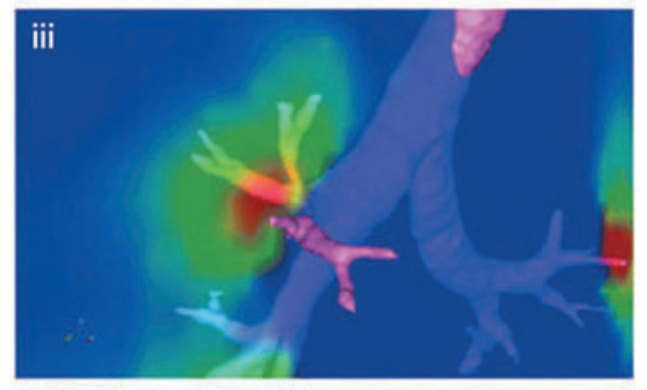

Air

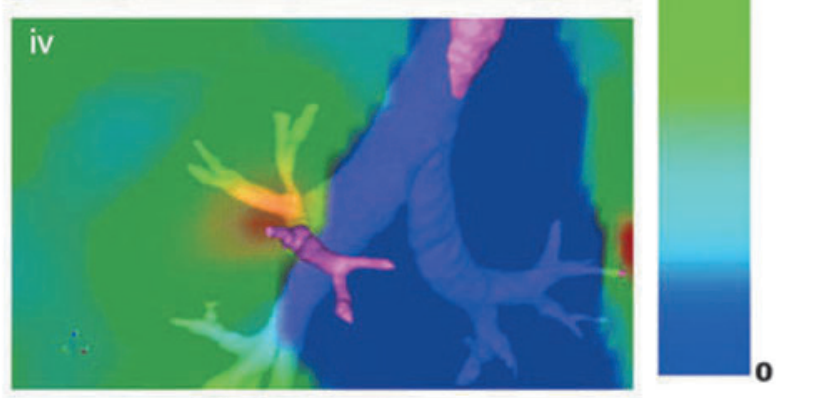

Helium-Oxygen

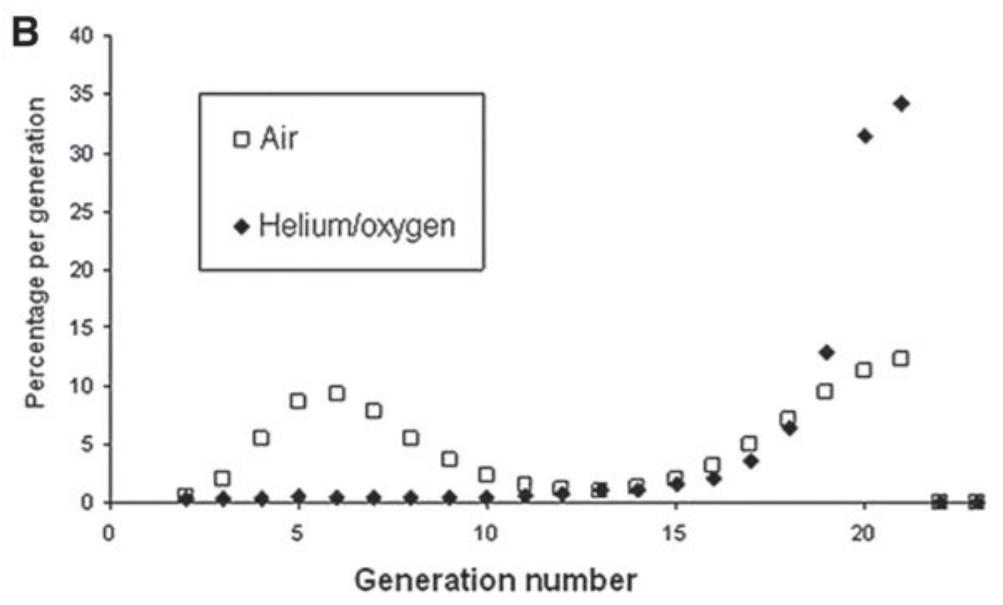

FIG. 4. (A) Deposition patterns obtained by 3D SPECT superimposed with segmented airways from high resolution CT on a background low resolution CT from an asthmatic subject breathing air (i,iii) or heliox (ii, iv) with particle size and inhalation pattern held constant. The scale on the right represents the percentage of the maximum deposition/voxel. (B) Estimated generational deposition fractions for air and heliox from the SPECT data for the same subject. Particle aerodynamic size was nominally $6 \mu \mathrm{m}$, and inhalation flow rate was $18 \mathrm{l} / \mathrm{min}$. Adapted from Katz and coworkers. ${ }^{(46)}$ Reproduced with permission from Mary Ann Liebert.

could be incorporated into formulation development for existing metered dose inhaler platforms, where similar phenomena have been noted. ${ }^{(32,54)}$

\section{Local Aerosol Targeting}

In the present context, local aerosol targeting is used to describe approaches that aim to target deposition to one or more specific, spatially defined locations within the respiratory tract. Such techniques are less developed than the passive targeting methods described above, and their use requires $a$ priori knowledge of target sites within the lungs. Accordingly, coordination with medical imaging can be assumed as an essential requirement. These are exploratory techniques that have potential use as research tools well ahead of clinical application. That said, even in a research setting, the ability to more precisely target aerosol delivery within the lungs to heterogeneously distributed sites of disease would enable investigations to improve understanding of the interplay between local, regional, and whole-lung doses with therapeutic effects and side effects. Three diverse approaches to local aerosol targeting are outlined below.

\section{Nebulizing catheters}

Conceptually, one of the simplest approaches to local aerosol targeting is to position the point of aerosol generation within the targeted region. This can be accomplished by 
positioning the tip of a nebulizing catheter in the target region using a bronchoscope. The catheter itself is essentially a long, thin tube containing lumen to convey pressurized air and liquid medication to its distal tip, where a spray of fine droplets is emitted. Localized aerosol delivery to specific lung lobes has been explored in this manner in animal models of lung cancer. ${ }^{(23,24)}$ While droplet deposition was primarily confined to the targeted lobe, some deposition did occur within non-targeted sites. ${ }^{(23)}$ Certainly, deposition proximal to the targeted lobe, in the trachea, likely occurred during exhalation, as aerosol was administered continuously over the breathing cycle. ${ }^{(23)}$ In this case, some redistribution of aerosol to non-targeted lobes may also occur during cycling from exhalation to inhalation. Nonetheless, nebulizing catheters do appear to offer a means to discriminately target aerosol deposition, in a parallel sense, between lung lobes. Within a selected lobe, the ability to target, in a serial sense, central versus peripheral airways remains to be explored.

\section{Controlled particle release position}

While nebulizing catheters have been used in preclinical studies, the invasiveness of the administration procedure when compared to use of conventional inhalers and nebulizers may limit clinical applications. The possibility that similar control over aerosol penetration into lung lobes or segments might be achieved through very precise manipulation of aerodynamic particle size, inhalation pattern, and the position of aerosol release at the proximal airway opening (e.g., at the mouthpiece) has been proposed by Kleinstreuer and colleagues. ${ }^{(55)}$ In principle, for a fixed airway geometry and steady, low inhalation flow rate, aerosol particles injected at a given location within the airway opening cross-section will follow similar paths during transit through the upper and central branching airways, arriving at similar downstream locations (provided they are sufficiently small aerodynamically to avoid deposition en route). Accordingly, there exists in theory a mapping between the particle release position and the distribution of aerosol penetration between parallel paths within the lungs. This is an intriguing concept, given that with conventional delivery approaches aerosol penetration will in general follow ventilation distribution. ${ }^{(56)}$ Controlling particle release position might be used to increase fractional penetration into poorly ventilated lung regions. However, in practice, the sensitivity of the mapping to small changes in flow rate needs to be assessed as does sensitivity to inevitable geometric changes resulting, for example, from movement of the jaw and tongue, tilt of the head, or repositioning the mouth-piece. Even with these variables held constant, determining optimal release locations to target a given downstream region for a given subject presents a significant challenge, most probably requiring computational simulation in airway geometries segmented from computed tomographic images, followed by validation with radiolabeled test aerosols in each individual subject. These obstacles to practical implementation may limit application of the technique in the foreseeable future.

\section{Magnetic targeting}

Varied approaches have been recently considered to locally target aerosol deposition using externally applied magnetic fields. ${ }^{(56-63)}$ As with other local targeting methods, the potential to deliver inhaled chemotherapeutic agents to sites of lung tumors is usually cited as motivation. Typically, this approach relies on generation of a magnetic body force on aerosol particles containing both drug and a magneticallyresponsive substance. ${ }^{(57-59,62,63)}$ Superparamagnetic iron oxide nanoparticles (SPIONs) are often used. Under the influence of an external magnetic field, these nanoparticles exhibit magnetization typical of their bulk ferromagnetic material (i.e., iron oxide). However, due to their very small size, they are composed of only a single magnetic domain, and therefore in the absence of an external magnetic field with no stabilization from neighboring domains, magnetization randomly flips direction at sufficiently high frequency to appear as an average of zero. The particles thus exhibit paramagnetic behavior but with much larger magnetization: hence, the moniker superparamagnetic. The magnetic body force exerted on a particle containing SPIONs will depend on the volume of SPIONs in the particle, the SPION magnetization, and the applied magnetic field gradient. ${ }^{(60)}$ The magnetization in turn is a function of the SPION volume magnetic susceptibility and magnetic field strength.

A considerable challenge that arises when using magnetic force for targeting drug delivery within the body is in the generation of high gradient magnetic fields at a substantial distance away from their source. Because the distance between target site and magnetic field source is generally much larger in humans or large animals than in small animals, experimental apparatus used to demonstrate positive results in small animals is often difficult to scale up. Still, it appears likely that magnetic forces at least on the order of the force of gravity can be generated on particles within the lungs using external permanent magnets, ${ }^{(60)}$ which may have utility in enhancing deposition in targeted peripheral lung regions. Much higher field gradients required for targeting central airway sites would require a purpose-built apparatus.

In contrast to the magnetic force, a magnetic torque is generated on non-spherical particles in uniform magnetic fields, with no gradient. High aspect ratio, rod-shaped aerosol particles will align with relatively low-strength fields, similar to the needle of a compass. Magnetic alignment of high aspect ratio particles has been shown to enhance deposition by interception in vitro in small airway models ${ }^{(60)}$ and to alter regional deposition patterns in vivo in an animal study. ${ }^{(61)}$ However, the deposition enhancement that can be achieved using this method of targeting appears to be inherently limited to a few multiples of baseline nontargeted values. ${ }^{(64)}$

A further aspect of magnetic targeting is the complimentary use of SPIONs as magnetic resonance imaging (MRI) contrast agents for in vivo measurement of regional lung deposition. ${ }^{(61,65-67)}$ This provides a potential tool for assessing targeting efficiency directly after administration, without need for any additional tracer or label. Use of MRI to measure aerosol deposition is discussed in detail elsewhere in this text.

\section{Summary}

- Targeting aerosol delivery to the lung by limiting upper airways deposition is a key consideration in the design of modern aerosol drug delivery devices. 
- Targeting within the lung to central or peripheral regions is likely to influence the effects of many inhaled therapies, though direct evidence to support this assumption is limited.

- Optimization of particle aerodynamic size is widely used in strategies to passively target central or peripheral lung regions.

- A number of additional techniques are available to further refine passive aerosol targeting, including control of inhalation pattern, bolus delivery, inhalation with heliox in place of air, and transient size manipulation using controlled evaporation and/or enhanced condensational growth.

- Local aerosol targeting to very specific, spatiallydefined lung regions is in its infancy, but several novel approaches are under exploration.

\section{Author Disclosure Statement}

The author has no conflicts of interest to disclose.

\section{References}

1. Langer R: Drug delivery and targeting. Nature. 1998; 392(6679 Suppl):5-10.

2. Torchilin VP: Passive and active drug targeting: Drug delivery to tumors as an example. Handb Exp Pharmacol. 2010;3-53.

3. Borgström L, Olsson B, and Thorsson L: Degree of throat deposition can explain the variability in lung deposition of inhaled drugs. J Aerosol Med. 2006;19:473-483.

4. Byron PR, Hindle M, Lange CF, Longest PW, McRobbie D, Oldham MJ, Olsson B, Thiel CG, Wachtel H, and Finlay WH: In vivo-in vitro correlations: Predicting pulmonary drug deposition from pharmaceutical aerosols. J Aerosol Med Pulm Drug Deliv. 2010;23 Suppl 2:S59-S69.

5. Finlay WH, and Martin AR: Recent advances in predictive understanding of respiratory tract deposition. J Aerosol Med Pulm Drug Deliv. 2008;21:189-206.

6. Grgic B, Finlay W, Burnell PK, and Heenan A: In vitro intersubject and intrasubject deposition measurements in realistic mouth-throat geometries. J Aerosol Sci. 2004;35: 1025-1040.

7. Olsson B, Borgström L, Lundbäck H, and Svensson M: Validation of a general in vitro approach for prediction of total lung deposition in healthy adults for pharmaceutical inhalation products. J Aerosol Med Pulm Drug Deliv. 2013; 26:355-369.

8. Tian G, Longest PW, Li X, and Hindle M: Targeting aerosol deposition to and within the lung airways using excipient enhanced growth. J Aerosol Med Pulm Drug Deliv. 2013;26: 248-265.

9. Patton JS, Brain JD, Davies LA, Fiegel J, Gumbleton M, Kim K-J, Sakagami M, Vanbever R, and Ehrhardt C: The particle has landed-Characterizing the fate of inhaled pharmaceuticals. J Aerosol Med Pulm Drug Deliv. 2010;23 Suppl 2:S71-S87.

10. Hultquist C, Wollmer P, Eklundh G, and Jonson B: Effect of inhaled terbutaline sulphate in relation to its deposition in the lungs. Pulm Pharmacol. 1992;5:127-132.

11. Zainudin BM, Biddiscombe M, Tolfree SE, Short M, and Spiro SG: Comparison of bronchodilator responses and deposition patterns of salbutamol inhaled from a pressurised metered dose inhaler, as a dry powder, and as a nebulised solution. Thorax. 1990;45:469-473.
12. Usmani OS, Biddiscombe MF, and Barnes PJ: Regional lung deposition and bronchodilator response as a function of beta2-agonist particle size. Am J Respir Crit Care Med. 2005;172:1497-1504.

13. Zanen P, Go LT, and Lammers J-WJ: The optimal particle size for $\beta$-adrenergic aerosols in mild asthmatics. Int $\mathbf{J}$ Pharm. 1994;107:211-217.

14. Zanen P: The optimal particle size for parasympathicolytic aerosols in mild asthmatics. Int J Pharm. 1995;114:111115.

15. Bakker EM, Volpi S, Salonini E, van der Wiel-Kooij EC, Sintnicolaas CJJCM, Hop WCJ, Assael B, Merkus P, and Tiddens H: Improved treatment response to dornase alfa in cystic fibrosis patients using controlled inhalation. Eur Respir J. 2011;38:1328-1335.

16. Bakker EM, Volpi S, Salonini E, Müllinger B, Kroneberg P, Bakker M, Hop W, Assael B, and Tiddens H: Small airway deposition of dornase alfa during exacerbations in cystic fibrosis; a randomized controlled clinical trial. Pediatr Pulmonol. 2014;49:154-161.

17. Laube BL.: The expanding role of aerosols in systemic drug delivery, gene therapy, and vaccination. Respir Care. 2005; 50:1161-1176.

18. Patton JS, and Byron PR.: Inhaling medicines: Delivering drugs to the body through the lungs. Nat Rev Drug Discov. 2007;6:67-74.

19. Bitonti AJ, Dumont JA, Low SC, Peters RT, Kropp KE, Palombella VJ, Stattel JM, Lu YC, Tan CA, Song JJ, Garcia AM, Simister NE, Spiekermann GM, Lencer WI, and Blumberg RS: Pulmonary delivery of an erythropoietin Fc fusion protein in non-human primates through an immunoglobulin transport pathway. Proc Natl Acad Sci U S A. 2004;101:9763-9768.

20. Bennett WD, Brown JS, Zeman KL, Hu S-C, Scheuch G, and Sommerer K.: Targeting delivery of aerosols to different lung regions. J Aerosol Med. 2002;15:179-188.

21. Ambrosini V, Nicolini S, Caroli P, Nanni C, Massaro A, Marzola MC, Rubello D, and Fanti S: PET/CT imaging in different types of lung cancer: An overview. Eur J Radiol. 2012;81:988-1001.

22. Darwiche K, Zarogoulidis P, Karamanos NK, Domvri K, Chatzaki E, Constantinidis TC, Kakolyris S, and Zarogoulidis K: Efficacy versus safety concerns for aerosol chemotherapy in non-small-cell lung cancer: A future dilemma for micro-oncology. Future Oncol. 2013;9:505-525.

23. Selting K, Waldrep JC, Reinero C, Branson K, Gustafson D, Kim DY, Henry C, Owen N, Madsen R, and Dhand R: Feasibility and safety of targeted cisplatin delivery to a select lung lobe in dogs via the AeroProbe intracorporeal nebulization catheter. J Aerosol Med Pulm Drug Deliv. 2008;21:255-268.

24. Selting K, Essman S, Reinero C, Branson KR, Henry CJ, Owen N, Guntur VP, Waldrep J, Kim DY, and Dhand R: Targeted combined aerosol chemotherapy in dogs and radiologic toxicity grading. J Aerosol Med Pulm Drug Deliv. 2011;24:43-48.

25. Stahlhofen W, Rudolf G, and James AC.: Intercomparison of experimental regional aerosol deposition data. J Aerosol Med. 1989;2:285-308.

26. Dolovich MA.: Influence of inspiratory flow rate, particle size, and airway caliber on aerosolized drug delivery to the lung. Respir Care. 2000;45:597-608.

27. Laube BL, Jashnani R, Dalby RN, and Zeitlin PL.: Targeting aerosol deposition in patients with cystic fibrosis: 
Effects of alterations in particle size and inspiratory flow rate. Chest. 2000;118:1069-1076.

28. Zeman KL, Wu J, and Bennett WD.: Targeting aerosolized drugs to the conducting airways using very large particles and extremely slow inhalations. J Aerosol Med Pulm Drug Deliv. 2010;23:363-369.

29. DeLong M, Wright J, Dawson M, Meyer T, Sommerer K, and Dunbar C.: Dose delivery characteristics of the AIR pulmonary delivery system over a range of inspiratory flow rates. J Aerosol Med. 2005; 18:452-459.

30. Duddu SP, Sisk SA, Walter YH, Tarara TE, Trimble KR, Clark AR, Eldon MA, Elton RC, Pickford M, Hirst PH, Newman SP, and Weers JG: Improved lung delivery from a passive dry powder inhaler using an engineered PulmoSphere ${ }^{\circledR}$ Powder. Pharm Res. 2002;19:689-695.

31. Hoe S, Traini D, Chan H-K, and Young PM.: The influence of flow rate on the aerosol deposition profile and electrostatic charge of single and combination metered dose inhalers. Pharm Res. 2009;26:2639-2646.

32. Shemirani FM, Hoe S, Lewis D, Church T, Vehring R, and Finlay WH.: In vitro investigation of the effect of ambient humidity on regional delivered dose with solution and suspension MDIs. J Aerosol Med Pulm Drug Deliv. 2013; 26:215-222.

33. Stein SW, and Myrdal PB.: The relative influence of atomization and evaporation on metered dose inhaler drug delivery efficiency. Aerosol Sci Technol. 2006;40:335347.

34. Lavorini F, Magnan A, Dubus JC, Voshaar T, Corbetta L, Broeders M, Dekhuijzen R, Sanchis J, Viejo JL, Barnes P, Corrigan C, Levy M, and Crompton GK: Effect of incorrect use of dry powder inhalers on management of patients with asthma and COPD. Respir Med. 2008;102:593-604.

35. Weers J, Ung K, Le J, Rao N, Ament B, Axford G, Maltz D, and Chan L: Dose emission characteristics of placebo PulmoSphere ${ }^{\circledR}$ particles are unaffected by a subject's inhalation maneuver. J Aerosol Med Pulm Drug Deliv. 2013; 26:56-68.

36. Denyer J, and Dyche T: The adaptive aerosol delivery (AAD) technology: Past, present, and future. J Aerosol Med Pulm Drug Deliv. 2010;23 Suppl 1:S1-S10.

37. Fischer A, Stegemann J, Scheuch G, and Siekmeier R: Novel devices for individualized controlled inhalation can optimize aerosol therapy in efficacy, patient care and power of clinical trials. Eur J Med Res. 2009;14 Suppl 4: 71-77.

38. Brand P, Meyer T, Häussermann S, Schulte M, Scheuch G, Bernhard T, Sommerauer B, Weber N, and Griese M: Optimum peripheral drug deposition in patients with cystic fibrosis. J Aerosol Med. 2005;18:45-54.

39. Clark AR, and Hartman MS: Regional lung deposition: Can it be controlled and have an impact on safety and efficacy? Respir Drug Deliv 2012. 2012;1:89-100.

40. Heinonen E, Meriläinen P, and Högman M: Administration of nitric oxide into open lung regions: Delivery and monitoring. Br J Anaesth. 2003;90:338-342.

41. Clark AR, Chambers CB, Muir D, Newhouse MT, Paboojian $S$, and Schuler C: The effect of biphasic inhalation profiles on the deposition and clearance of coarse $(6.5$ microm) bolus aerosols. J Aerosol Med. 2007;20:75-82.

42. Anderson M, Svartengren M, Bylin G, Philipson K, and Camner P: Deposition in asthmatics of particles inhaled in air or in helium-oxygen. Am Rev Respir Dis. 1993;147: 524-528.
43. Conway J, Fleming J, Majoral C, Katz I, Perchet D, Peebles C, Tossici-Bolt L, Collier L, Caillibotte G, Pichelin M, Sauret-Jackson V, Martonen T, Apiou-Sbirlea G, Muellinger B, Kroneberg P, Gleske J, Scheuch G, Texereau J, Martin A, Montesantos S, and Bennett M: Controlled, parametric, individualized, 2-D and 3-D imaging measurements of aerosol deposition in the respiratory tract of healthy human subjects for model validation. J Aerosol Sci. 2012;52: $1-17$.

44. Corcoran TE, Shortall BP, Kim IK, Meza MP, and Chigier $\mathrm{N}$ : Aerosol drug delivery using heliox and nebulizer reservoirs: Results from an MRI-based pediatric model. J Aerosol Med. 2003;16:263-271.

45. Darquenne $\mathrm{C}$, and Prisk GK: Aerosol deposition in the human respiratory tract breathing air and 80:20 heliox. J Aerosol Med. 2004;17:278-285.

46. Katz I, Pichelin M, Montesantos S, Majoral C, Martin A, Conway J, Fleming J, Venegas J, Greenblatt E, and Caillibotte G: Using helium-oxygen to improve regional deposition of inhaled particles: Mechanical principles. J Aerosol Med Pulm Drug Deliv. 2014;27:71-80.

47. Peterson JB, Prisk GK, and Darquenne C: Aerosol deposition in the human lung periphery is increased by reduceddensity gas breathing. J Aerosol Med Pulm Drug Deliv. 2008;21:159-168.

48. Javaheri E, Shemirani FM, Pichelin M, Katz IM, Caillibotte G, Vehring R, and Finlay WH: Deposition modeling of hygroscopic saline aerosols in the human respiratory tract: Comparison between air and helium-oxygen as carrier gases. J Aerosol Sci. 2013;64:81-93.

49. Martin AR, Gleske J, Katz IM, Hartmann M, Mullinger B, Haussermann S, Caillibotte G, and Scheuch G: Laser diffraction characterization of droplet size distributions produced by vibrating mesh nebulization in air and a heliumoxygen mixture. J Aerosol Sci. 2010;41:1159-1166.

50. Hindle M, and Longest PW: Condensational growth of combination drug-excipient submicrometer particles for targeted high-efficiency pulmonary delivery: Evaluation of formulation and delivery device. J Pharm Pharmacol. 2012; 64:1254-1263.

51. Longest PW, and Hindle M: CFD simulations of enhanced condensational growth (ECG) applied to respiratory drug delivery with comparisons to in vitro data. J Aerosol Sci. 2010;41:805-820.

52. Javaheri E, and Finlay WH: Size manipulation of hygroscopic saline droplets: Application to respiratory drug delivery. Int J Heat Mass Transf. 2013;67:690-695.

53. Golshahi L, Tian G, Azimi M, Son Y-J, Walenga R, Longest PW, and Hindle M: The use of condensational growth methods for efficient drug delivery to the lungs during noninvasive ventilation high flow therapy. Pharm Res. 2013;30:2917-2930.

54. Martin AR, and Finlay WH: The effect of humidity on the size of particles delivered from metered-dose inhalers. Aerosol Sci Technol. 2005;39:283-289.

55. Kleinstreuer C, Zhang Z, and Donohue JF: Targeted drugaerosol delivery in the human respiratory system. Annu Rev Biomed Eng. 2008;10:195-220.

56. Darquenne $\mathrm{C}$, van Ertbruggen $\mathrm{C}$, and Prisk GK: Convective flow dominates aerosol delivery to the lung segments. J Appl Physiol. 2011;111:48-54.

57. Ally J, Martin B, Behrad Khamesee M, Roa W, and Amirfazli A: Magnetic targeting of aerosol particles for cancer therapy. J Magn Magn Mater. 2005;293:442-449. 
58. Dames P, Gleich B, Flemmer A, Hajek K, Seidl N, Wiekhorst F, Eberbeck D, Bittmann I, Bergemann C, Weyh T, Trahms L, Rosenecker J, and Rudolph C: Targeted delivery of magnetic aerosol droplets to the lung. Nat Nanotechnol. 2007;2:495-499.

59. Hasenpusch G, Geiger J, Wagner K, Mykhaylyk O, Wiekhorst F, Trahms L, Heidsieck A, Gleich B, Bergemann C, Aneja MK, and Rudolph C: Magnetized aerosols comprising superparamagnetic iron oxide nanoparticles improve targeted drug and gene delivery to the lung. Pharm Res. 2012;29:1308-1318.

60. Martin AR, and Finlay WH: Enhanced deposition of high aspect ratio aerosols in small airway bifurcations using magnetic field alignment. J Aerosol Sci. 2008;39:679-690.

61. Redman GE, Martin AR, Waszak P, Thompson RB, Cheung PY, and Thebaud B: Pilot study of inhaled aerosols targeted via magnetic alignment of high aspect ratio particles in rabbits. J Nanomater. 2011;2011.

62. Upadhyay D, Scalia S, Vogel R, Wheate N, Salama RO, Young PM, Traini D, and Chrzanowski W: Magnetised thermo responsive lipid vehicles for targeted and controlled lung drug delivery. Pharm Res. 2012;29:2456-2467.

63. Xie Y, Longest PW, Xu YH, Wang JP, and Wiedmann TS: In vitro and in vivo lung deposition of coated magnetic aerosol particles. J Pharm Sci. 2010;99:4658-4668.

64. Martinez RC, Roshchenko A, Minev P, and Finlay WH: Simulation of enhanced deposition due to magnetic field alignment of ellipsoidal particles in a lung bifurcation. J Aerosol Med Pulm Drug Deliv. 2013;26:31-40.
65. Martin AR, Thompson RB, and Finlay WH: MRI measurement of regional lung deposition in mice exposed nose-only to nebulized superparamagnetic iron oxide nanoparticles. J Aerosol Med Pulm Drug Deliv. 2008;21:335-342.

66. Oakes JM, Scadeng M, Breen EC, Prisk GK, and Darquenne C: Regional distribution of aerosol deposition in rat lungs using magnetic resonance imaging. Ann Biomed Eng. 2013;41:967-978.

67. Thompson RB, and Finlay WH: Using MRI to measure aerosol deposition. J Aerosol Med Pulm Drug Deliv. 2012; 25:55-62.

Address correspondence to:

Andrew R. Martin, PhD, PEng

10-324 Donadeo Innovation Center for Engineering

University of Alberta

Edmonton, Alberta

T6G 1 H9 Canada

E-mail: andrew.martin@ualberta.ca

Abbreviations
COPD $=$ chronic obstructive pulmonary disease
MRI $=$ magnetic resonance imaging
SPIONs $=$ superparamagnetic iron oxide nanoparticles

